\title{
Carry-over effects of soil inoculation on plant growth and health under sequential exposure to soil-borne diseases
}

\author{
Hai-kun Ma (iD) Ana Pineda • Andre W. G. van der Wurff • \\ T. Martijn Bezemer
}

Received: 21 June 2018 / Accepted: 2 October 2018 / Published online: 25 October 2018

(C) The Author(s) 2018

\begin{abstract}
Background and aims Most plant-soil feedback and inoculation studies are limited to one growth cycle. We examined the effects of inoculation with eight plantconditioned soils on chrysanthemum during two sequential growth cycles. The plants were also exposed sequentially to soil diseases.

Methods In cycle 1, plants were grown in sterile soil inoculated or not with plant-conditioned soils, and exposed or not to Pythium or root feeding nematodes. In cycle 2, new plants were grown in soil from cycle 1 or in new $100 \%$ sterile soil. Plants were exposed again to Pythium, or to soil with pathogens and nematodes collected from a commercial chrysanthemum greenhouse.
\end{abstract}

Responsible Editor: Matthew G. Bakker.

Electronic supplementary material The online version of this article (https://doi.org/10.1007/s11104-018-3837-9) contains supplementary material, which is available to authorized users.

H.-k. Ma · A. Pineda • T. M. Bezemer

Department of Terrestrial Ecology, Netherlands Institute of Ecology (NIOO-KNAW), P.O. Box 50, 6700 ABWageningen, The Netherlands

H.-k. Ma $(\bowtie) \cdot$ T. M. Bezemer

Institute of Biology, Section Plant Ecology and Phytochemistry, Leiden University, P.O. Box 9505, 2300 RALeiden,

The Netherlands

e-mail: H.Ma@nioo.knaw.nl

A. W. G. van der Wurff

Delft Research Group, Section Green Projects, Groen Agro

Control, P.O. Box 549, 2600 AMDelft, The Netherlands
Results After two cycles, effects of soil inoculation on plant growth were still present. Chrysanthemum exhibited a negative conspecific feedback response, but this was less strong in inoculated soils. Pythium or nematode addition did not affect plant growth. However, addition of pathogen-containing soil from the commercial greenhouse reduced plant growth in sterile soil but increased growth in plant-conditioned soils.

Conclusions Inoculation with plant-conditioned soil can reduce the negative conspecific plant-soil feedback of chrysanthemum. Our study further advances our understanding of the temporal dynamics of conspecific and heterospecific plant-soil feedbacks, and how they interact with soil-borne diseases.

Keywords Carry-over effects · Soil inoculation . Chrysanthemum $\cdot$ Soil-borne disease $\cdot$ Plant-soil feedback

\section{Introduction}

Belowground plant pathogens, parasites, herbivores and mutualists can greatly influence the performance of plants (Wardle et al. 2004). Plants also selectively affect soil biota by releasing organic compounds into the soil via e.g. root exudation or dead plant material (Wardle et al. 2004). This interdependency leads to feedbacks between plants that grew first in the soil and plants that grow later in that soil, a phenomenon called plant-soil feedback (van der Putten et al. 2013; Bever et al. 1997). Many plant species grow better in soil where previously 
another plant species was grown than in their own soil (Kulmatiski et al. 2008; van de Voorde et al. 2011; Cortois et al. 2016). There is increasing interest to implement soil ecological concepts such as plant-soil feedbacks into agricultural systems to enhance soil health and quality and therefore the sustainability of crop production (Pineda et al. 2017; Mariotte et al. 2017; Zhou et al. 2017; Wang et al. 2017a). It is wellknown that inoculation with specific soil microbes can reduce the susceptibility of a crop to pests and diseases. However, a number of studies have reported that many of these disease-suppressive strains poorly colonize and survive in the rhizosphere (e.g. Gómez Expósito et al. 2017; Alabouvette et al. 2009). Inoculation of soil with beneficial microbiomes rather than with individual microbial species can also transfer disease-suppressive properties, and may be more efficient than inoculation with specific microbial strains (Pineda et al. 2017; Schlatter et al. 2017; Ma et al. 2017; Chaparro et al. 2012). An important challenge is to examine how long these soil community inocula remain effective, and thus whether we can improve crop health in the longer term using heterospecific plant-soil feedback principles.

The biotic and abiotic legacies that plants leave in the soil can have long-lasting effects on other plants, and this has been detected both in natural and agricultural systems (Kulmatiski et al. 2006, Kulmatiski and Beard 2011; Detheridge et al. 2016). These long-term soil mediated effects could be due to the persistence of plant allelochemicals in the soil (Huang et al. 2013), or due to long dormancy stages of certain soil organisms (Lennon and Jones 2011), or because many free-living soil microbes can survive in the soil without their host (Lapsansky et al. 2016). Recently, two microcosm studies showed that after sequentially growing different plant species in the same soil, the first plant species, via a legacy left in the soil, still influenced a third plant species even though another plant species had been grown in the soil intermediately (Wubs and Bezemer 2018; Bezemer et al. 2018). However, the plant that grew most recently in the soil had the strongest influence, via its effect on the soil, on the last plant (Wubs and Bezemer 2018). Thus, by repeatedly growing a focal plant in soil inoculated with a microbial community, we would expect that the impact of the microbial community that was inoculated at the beginning may still be detected in later growth cycles. However, we also expect that the influence of the focal plant itself on later plant growth (i.e. conspecific plantsoil feedback) will increase with time. Hence, the effect of the microbial community that was inoculated initially will diminish over time (Bezemer et al. 2018).

How carry-over effects of inoculation on plant growth are influenced by exposure of the focal plant to soil-borne diseases is unknown. Several studies have shown that repeated exposure to soil pathogens during different growth cycles affects plant growth more negatively than a single exposure to soil pathogens (Hajihassani et al. 2013; Khan and Siddiqui 2017; Whitelaw-Weckert et al. 2013). In such sequential interactions between plants and belowground pathogenic organisms, the first inoculation with a pathogen may have a stronger negative influence on plant growth than the second inoculation (Siddiqui et al. 1999; Castillo et al. 1998; Wurst and Ohgushi 2015; Pung et al. 1991). However, the severity of sequential inoculations with pathogens will also depend on the microbial community that is present. For example, microbial communities that negatively affect the growth of a focal plant may also increase the susceptibility of this plant to other soilborne diseases, while soil microbial communities that suppress soil pathogens, may reduce the susceptibility of the focal plant to later exposure to (other) soil pathogens (Mallon et al. 2018).

In this study, we examine how inoculation with soil microbial communities from eight plant species influences the growth and disease susceptibility of chrysanthemum during two growth cycles with sequential exposure to different soil-borne diseases. The focal plant chrysanthemum (Dendrathema X grandiflora) is a commercial cut-flower, and in commercial greenhouses in the Netherlands, the soil is sterilized regularly by steaming to control soil pathogens (Thuerig et al. 2009; Tamm et al. 2010). The selection of the eight plant species used in this study to condition the soil was based on a previous study, in which we observed that inoculation with plant-conditioned soil had highly variable effects on chrysanthemum performance and that the effects were species-specific (Ma et al. 2017). Based on these previous results, we selected plant species with positive and negative effects on chrysanthemum, to examine how these positive and negative soil feedback effects develop when chrysanthemum is also sequentially exposed to soil-borne diseases. We exposed plants in inoculated and un-inoculated soil to the root pathogen Pythium ultimum and the root knot nematode Meloidogyne incognita in the first growth cycle. Pythium ultimum is an important root pathogen in chrysanthemum and causes symptoms such as root rot 
(Reddy 2015; Pettitt et al. 2011). Meloidogyne incognita is a sedentary root endoparasite, which causes root galls in chrysanthemum and subsequent leaf yellowing, eventually resulting in stunted growth (Johnson and Littrell 1970; Siddiqui et al. 2014). In the second growth cycle, we grew new chrysanthemum plants in the soil from cycle 1. These plants were exposed again to Pythium ultimum or to $10 \%$ "diseased soil" collected from a commercial chrysanthemum greenhouse with a severe Meloidogyne infestation. We tested four hypotheses: 1) the effects of inoculation at the beginning of the first growth cycle will remain present in the second growth cycle; but 2) negative conspecific feedback effects of chrysanthemum will more strongly influence plant growth in the second cycle than the effects of initial inoculation with plant-conditioned soil; and 3) inoculation with plant-conditioned soils that have negative effects on chrysanthemum will increase the negative effects of introduced soil-borne diseases in the second growth cycle, while plant-conditioned soil inocula with positive effects on chrysanthemum growth will suppress the effects of soil-borne diseases in the second growth cycle. 4) Plant growth in the second cycle will be more strongly influenced by soil-borne diseases added in the first than in the second growth cycle.

\section{Materials and methods}

Plant material

The focal plant in our study was Dendranthema $X$ grandiflora (Ramat.) Kitam. cv. Grand Pink (Chrysanthemum, syn. Chrysanthemum X morifolium (Ramat.) Hemsl., Asteraceae). Chrysanthemum cuttings were provided by the breeding company FIDES by Dümmen Orange (De Lier, The Netherlands).

\section{Pathogen propagations}

The soil-borne oomycete pathogen Pythium ultimum (Pythiaceae) was obtained from Wageningen UR Greenhouse Horticulture (Wageningen UR, Greenhouse Horticulture, Bleiswijk, The Netherlands). Pythium ultimum was isolated from chrysanthemum plants, and cultured on liquid V8 medium (200 ml of organic tomato suspension without added salt, $2 \mathrm{~g} \mathrm{CaCO}_{3}$, and $800 \mathrm{ml}$ water) at room temperature for 2 weeks. The P. ultimum culture was then blended in a mixer and filtered to obtain a solution with only oospores based on a modified protocol of van der Gaag and Wever (2005). The oospores concentration was determined by counting the number of oospores in $1 \mathrm{ml}$ liquid suspension under the microscope using a Fuchs-Rosenthal chamber.

Meloidogyne incognita J2 nematodes were obtained from HZPC Holland B.V., The Netherlands. The purity of the culture was assessed with speciesspecific markers by AgroXpertus (Wageningen, The Netherlands). The culture contained 99\% M. incognita and $1 \%$ M. hapla.

Diseased soil was obtained from a commercial chrysanthemum greenhouse in Made, The Netherlands. The soil from this commercial greenhouse contained high densities of Meloidogyne incognita.

\section{Experimental set-up}

The experiment consisted of three phases. In the conditioning phase, eight plant species were used to condition soil individually: Anthoxanthum odoratum, Poaceae (AO), Bromus hordeaceus, Poaceae (BH), Festuca filiformis, Poaceae (FF), Lolium perenne, Poaceae (LP), Holcus lanatus, Poaceae (HL), Rumex acetosella, Polygonaceae (RA), Galium verum, Rubiaceae (GV) and Hypochaeris radicata, Asteraceae (HR). For the next two growth cycles, chrysanthemum plants were grown repeatedly either in sterile soil inoculated with plant-conditioned soil or in sterile soil (un-inoculated), and either with exposure to different disease treatments in each cycle or not exposed (control). Disease treatments added in cycle 1 (Pythium or Meloidogyne or control) were termed as "disease 1 ", disease treatments added in cycle 2 (Pythium or diseased soil inoculum or control) were termed as "disease 2 ". The soils that were used at the start of cycle 1 as inoculum and conditioned by plant monocultures were termed "plant-conditioned inocula".

In cycle 2 , there were nine combined disease treatments, as a result of the full factorial combination of treatments in the first and the second cycle. Treatments were abbreviated with codes consisting of two letters, the first one represents the disease treatment applied during the first cycle and the second one represents the treatment imposed during the second cycle: control control (C-C), control - Pythium (C-P), control - diseased soil inoculum (C-D), Pythium - control (P-C), Pythium-Pythium (P-P), Pythium - diseased soil 
inoculum (P-D), Meloidogyne - control (M-C), Meloidogyne - Pythium (M-P), Meloidogyne - diseased soil inoculum (M-D). In addition, in cycle 2, the three disease treatments (control, Pythium, diseased soil) were also imposed to new sterilized soil making a total of 12 treatments in cycle 2. A schematic drawing of the experiment is presented in Fig. 1.

\section{Phase I: Conditioning phase}

For the conditioning phase, soil was collected $(5-20 \mathrm{~cm}$ deep) in June 2015 from a semi-natural grassland on former arable land (Mossel, Ede, The Netherlands). The field had been used for agricultural purpose until 1996. The sandy-loam soil was homogenized and sieved $(1 \mathrm{~cm}$ mesh size) to remove coarse fragments and all macroarthropods. Pots $(13 \times 13 \times 13 \mathrm{~cm})$ were filled with a homogenized mixture of field soil and sterilized field soil in a 1:1 ratio. Soil sterilization was done by gamma irradiation (> $25 \mathrm{~K}$ Gray gamma irradiation, Isotron, Ede, The Netherlands). The sterilized soil was added to minimize potential differences in soil nutrient heterogeneity and to provide a niche for the soil microbes to grow and hence increase the potential for plant-speciesspecific effects on the soil community. Pots were filled with $1.6 \mathrm{Kg}$ of soil (based on dry weight).
Seeds of all wild plant species were obtained from a wild plant seed supplier (Cruydt-Hoeck, Assen, The Netherlands). Seeds were surface sterilized in $3 \%$ sodium hypochlorite solution for $1 \mathrm{~min}$, rinsed and germinated on sterile glass beads in a climate chamber at $20^{\circ} \mathrm{C}$ (16 h/8 h, light/dark). Five one-week-old seedlings were transplanted in monocultures in each pot, and there were ten replicate pots for each species. In total, the conditioning phase comprised of 80 pots (monocultures of 8 plant species $\times 10$ replicates). Seedlings that died during the first week of the experiment were replaced. As a few seedlings died later, the number of seedlings in each pot was reduced to four so that the density was the same in all pots. All pots were placed randomly in a climate controlled greenhouse with $70 \% \mathrm{RH}, 16 \mathrm{~h}$ at $21^{\circ} \mathrm{C}$ (day) and $8 \mathrm{~h}$ at $16^{\circ} \mathrm{C}$ (night). Natural daylight was supplemented by $400 \mathrm{~W}$ metal halide lamps $\left(225 \mu \mathrm{mol} \mathrm{s}{ }^{-1} \mathrm{~m}^{-2}\right.$ photosynthetically active radiation, one lamp per $1.5 \mathrm{~m}^{2}$ ). The pots were watered regularly. Ten weeks after transplanting, the plants were carefully removed from each pot and the largest roots were removed from the soil as they may act as a source for regrowing plants. Finer roots were left in the soil as the rhizosphere around these roots may include a major part of the microbial rhizosphere community. The soil from each pot was homogenized and stored separately in plastic bags at $4{ }^{\circ} \mathrm{C}$ until used in the test phase so that there were 10 replicate soils for each plant species.

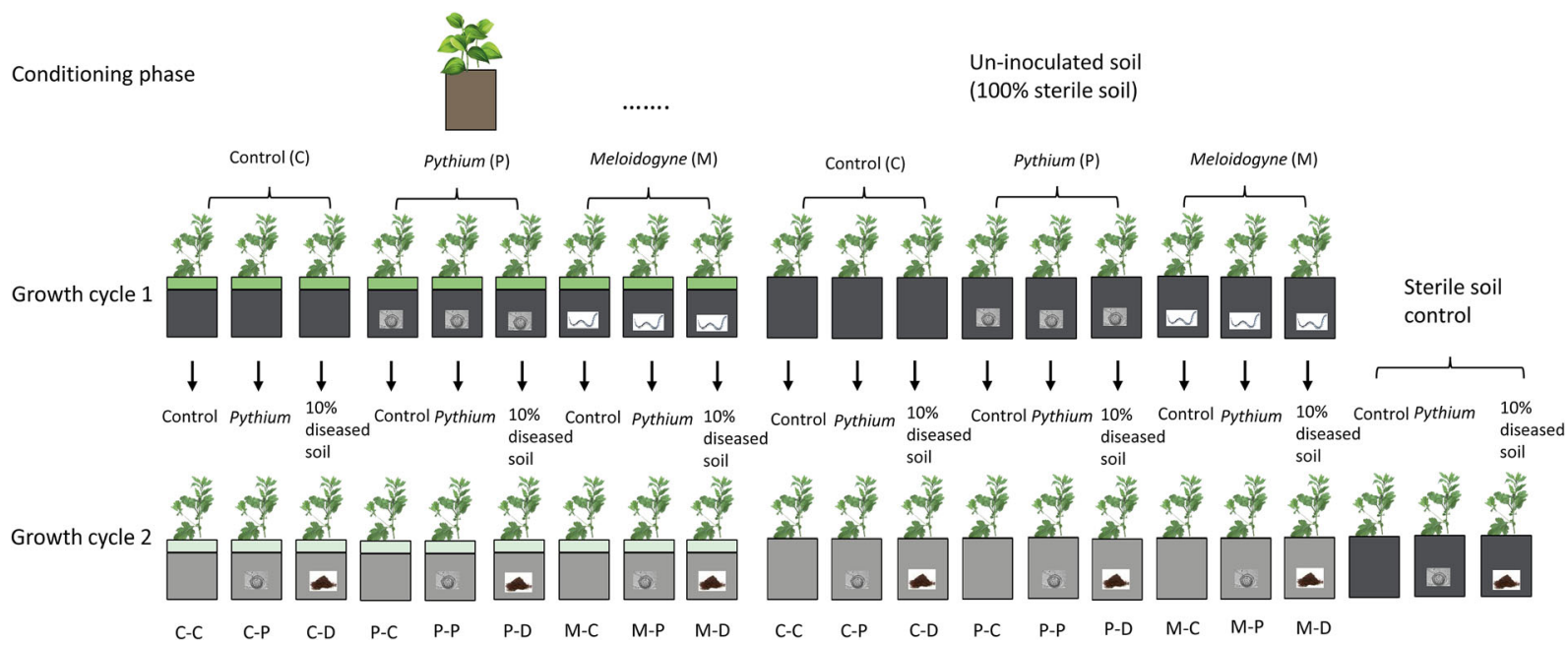

Fig. 1 Experimental design. Eight plant species were grown in monocultures for 10 weeks, for clarity, only one of the eight species is depicted. In growth cycle 1 , chrysanthemum was planted in $90 \%$ sterile soil inoculated with $10 \%$ plant conditioned soil. A set of replicates with un-inoculated soil $(100 \%$ sterile soil) was also included. Each soil treatment was further divided in three disease treatments: Pythium,

Meloidogyne or control. In growth cycle 2, all soils from the previous cycle were used for a second round of chrysanthemum growth. Each treatment combination from cycle 1 was divided into pots receiving Pythium, 10\% diseased soil inoculum, or control pots. In cycle 2, a new set of replicates with $100 \%$ sterile soil was included and these were also imposed to disease treatments 
Phase II: Growth cycle 1

For growth cycle $1,1 \mathrm{~L}$ pots $(11 \times 11 \times 12 \mathrm{~cm}$; length $\times$ wide $\times$ height) were filled with a homogenized mixture of $10 \%$ soil inoculum (plant species-specific conditioned soil) and $90 \%$ sterilized soil (see above). Pots filled with $100 \%$ sterilized soil served as control (uninoculated soil). Two chrysanthemum cuttings (without roots) were planted in each pot as preliminary work showed that not all cuttings establish properly with this method. Prior to planting, the soil in each pot was watered and $100 \mathrm{ml}$ half-strength Hoagland nutrient solution was added. The pots were placed on trolleys, each trolley had 48 pots and was tightly covered with a thin transparent plastic film for 10 days to create a closed environment with high humidity that favors rooting. After 10 days, one of the chrysanthemum cuttings was removed from each pot. Seven days after the transparent plastic film had been removed, $2 \mathrm{ml}$ of the oospore suspension (ca. 60,000 oospores of P. ultimum) was added onto the soil next to the stem of each plant allocated to the Pythium treatment. A $1.5 \mathrm{~cm}$ deep hole was made in the soil a near the stem of each plant allocated to the nematode treatment, and $5 \mathrm{ml}$ suspension containing $M$. incognita (ca. 5900 Juveniles stage 2 ) was added. Plants were fertilized following common practices used by chrysanthemum growers: halfstrength Hoagland nutrient solution for the first two weeks, single-strength Hoagland solution during the following two weeks. The strength was increased to $1.6 \mathrm{mS} / \mathrm{cm}$ EC (electrical conductivity) for the last two weeks. The density of pots on each trolley was reduced two weeks after the start of the second phase to 32 pots per trolley so that there was $10 \mathrm{~cm}$ space between each pot. There were three replicate pots for each soil from the conditioning phase. Hence, cycle 1 comprised of 810 pots $(8$ plant species-specific soil inocula $\times 3$ disease treatments $\times 10$ soil replicates $\times 3$ pot replicates + non-inoculated soil $\times 3$ disease treatments $\times 10$ soil replicates $\times 3$ pot replicates). All pots were randomly arranged in a greenhouse compartment and kept under the same conditions as described for the conditioning phase.

Six weeks after rooting, all plants were harvested. Plants were clipped at soil level and roots were removed from the soil and the soil was returned to each pot for the next grow cycle. Roots were washed over a sieve ( $2 \mathrm{~mm}$ mesh). For each plant, leaf yellowness was recorded as a plant health indicator, because in chrysanthemum leaf yellowness is symptomatic for diseases such as those caused by soil pathogens like Verticillium, Fusarium (Reddy 2015). All leaves were counted on each plant and the number of leaves that showed yellowness (partly, or completely) was recorded. Leaf yellowness was calculated as the proportion of leaves that showed yellowness. Root color was also recorded at a scale of 0 to 3 , where 3 indicates a dark and diseased root system, and 0 indicates a white/light colored and healthy root system (photos of root systems illustrative for the root darkness categories are presented in Fig.S1). Root galls caused by root knot nematodes were scored with a scale of 0 to 10 (Dhandaydham et al. 2008). $0=$ no visual galling, $1=$ less than $5 \%$ small galls, $2=10 \%$ small galls, $3=15 \%$ small galls, $4=20 \%$ large galls, $5=30 \%$ large galls, $6=40 \%$ big galls, $7=50 \%$ big galls, $8=60 \%$ big galls, $9=70 \%$ big galls, $10=$ more than $75 \%$ big galls. In cycle 1 , nematode scores were only recorded for chrysanthemum roots from plants exposed to the Meloidogyne treatment, but in cycle 2, this was done for all plants from all treatments. Shoot and root biomass was then oven-dried $\left(60^{\circ} \mathrm{C}\right.$ for 3 days) and weighed.

\section{Phase III: Growth cycle 2}

The experimental procedure was as described for cycle 1 . In this phase, we used as disease treatments P. ultimum addition $\left(6 \times 10^{\wedge} 4\right.$ oospores $), 10 \%$ "diseased" soil, i.e. soil collected from a commercial chrysanthemum greenhouse with severe soil disease problems, and control. Two new unrooted chrysanthemum cuttings were planted into each pot from growth cycle 1 as described above. A new set of 30 pots filled with $100 \%$ sterilized soil was included during growth cycle 2 , either with $10 \%$ diseased soil, or 60,000 oospores of $P$. ultimum, or control. This phase comprised of the same 810 pots plus 10 replicates for each of the 3 soil disease treatments using new $100 \%$ sterile soil, resulting in a total of 840 pots. Six weeks after rooting, plants were harvested as described above.

\section{Statistical analysis}

After growth cycle 1, the overall effects of the plantconditioned soil inocula and disease treatments on chrysanthemum shoot biomass and root biomass were determined using a linear mixed model. In the model, "soil identity" and "disease treatment 1 " were used as fixed 
factors, and soil replicate was used as random factor. Post-hoc Tukey tests were used for pairwise comparisons between plant-conditioned soil inocula. The same analysis was also performed including un-inoculated soil. A post-hoc Dunnet test was used to compare chrysanthemum biomass for each of the plant-conditioned soil inocula with that of un-inoculated soil. As chrysanthemum leaf yellowness is proportional data, a generalized linear mixed model with a binomial distribution and logit link function was used. Data on root darkness are categorical, therefore a generalized linear mixed model with Poisson distribution was used. Nematode scores were only recorded for plants in the Meloidogyne treatment, therefore, a generalized linear model was used to determine the overall effects of the soil inoculation treatments on nematode infection.

For data from growth cycle 2, the effects of the plantconditioned soil inocula and disease treatments on chrysanthemum shoot biomass and root biomass were determined using a linear mixed model. In the model, "soil identity", "disease treatment 1 " and "disease treatment 2 " were used as fixed factors, and soil replicate was used as random factor. A post-hoc Tukey test was used for pairwise comparisons among the disease treatments of both cycles. For root darkness and root nematode scores, a generalized linear mixed model with Poisson distribution was used to examine the overall soil effects (analyzed separately with and without un-inoculated soil) and effects of disease treatments in cycle 1 and disease treatments in cycle 2.

We then compared the effects of the three disease treatments imposed during cycle 2 on chrysanthemum performance in (i) soils inoculated in cycle 1, (ii) soils that were not inoculated in cycle 1 , and (iii) new sterile soil. We used the average for the inoculated soils as the differences between the effects of plant-conditioned inocula on chrysanthemum in cycle 2 were less variable compared with their effects in cycle 1 (see results). For inoculated and un-inoculated soils from cycle 1, there were 9 combinations of disease treatments ( 3 for cycle $1 \times 3$ for cycle 2 ) while for new sterile soil there were only 3 treatments ( 3 for cycle 2 ). The disease treatments added to inoculated and un-inoculated soil in cycle 1 were therefore grouped based on the three disease treatments from cycle 2. C-C, P-C and M-C of inoculated and un-inoculated soil were compared to the $\mathrm{C}$ in new sterile soil; C-P, P-P and M-P were compared to P, and C-D, P-D and M-D to D. One-way ANOVA was then used to compare these seven groups and a post-hoc
Tukey test was used for pairwise comparisons. These analyses were done for chrysanthemum shoot biomass, root biomass and leaf yellowness. As leaf yellowness were recorded as a proportion, a generalized linear model was used instead.

Additionally, chrysanthemum shoot biomass, root biomass and yellowness were grouped based on the soil treatment (soils inoculated in cycle 1 and soils that were not inoculated in cycle 1), and a linear mixed model was then used to examine the impact of the disease treatments. In this model, the disease treatments were used as fixed factor, and soil replicate was used as random factor. For sterile soil, one-way ANOVA was used to examine the impact of disease treatments. Within each disease treatment, chrysanthemum shoot and root biomass of plants growing in different inoculated soil was compared using one-way ANOVA. The same analysis was done for chrysanthemum leaf yellowness, but either with a generalized linear model or a generalized linear mixed model with binomial distribution and logit link function. To determine the effects of the disease treatments within each soil, a generalized linear model was used to compare chrysanthemum root darkness and root nematode score between different disease treatments. We also used linear regression to determine the relationship between root biomass of conditioning plant species and root biomass of chrysanthemum grown later in the conditioned soils. This analysis was done for root biomass of chrysanthemum in both cycle 1 and cycle 2 . All analyses were performed in $\mathrm{R}$ (version 3.0.1, $\mathrm{R}$ Development Core Team 2017).

\section{Results}

After growth cycle 1, chrysanthemum shoot biomass, root biomass and leaf yellowness significantly differed between plant-conditioned inocula, while "disease treatment 1" only significantly influenced leaf yellowness. Root darkness and the root nematode scores were not significantly affected by any of the treatments (Table 1 , Table S1, Fig. S2). When un-inoculated soil was included in the analysis, the same effects were significant (Table S1). Plants grown with AO inoculum sustained higher shoot and root biomass than plants with HR inoculum, and $\mathrm{AO}, \mathrm{BH}, \mathrm{GV}$, and LP inocula sustained significantly higher shoot biomass than un-inoculated soil (Fig. 2a, b). Root biomass of plants grown with plant-conditioned inocula did not differ from root 
Table 1 Effects of plant-conditioned soil inocula and disease treatment 1 on chrysanthemum shoot biomass, root biomass and leaf yellowness at the end of cycle 1. "Species" indicates the identity of the conditioning plant species. "Treatment 1 " indicates the disease treatments in cycle 1. Presented are F-values obtained from a mixed linear model (generalized mixed linear model for leaf yellowness). $* * *$ indicates significant difference at $P<0.001$

\begin{tabular}{lllll}
\hline & Df & Shoot biomass & Root biomass & Yellowness \\
\hline Species & 7,72 & $7.751 * * *$ & $4.678 * * *$ & $5.708 * * *$ \\
Treatment1 & 2624 & 1.101 & 0.263 & $8.240 * * *$ \\
Treatment $1 *$ Species & 14,624 & 1.472 & 1.113 & $5.694 * * *$ \\
\hline
\end{tabular}

biomass in un-inoculated soil (Fig. 2b). In the control, without addition of diseases, the proportion of yellow leaves, was significantly lower with $\mathrm{AO}, \mathrm{BH}$, or $\mathrm{HL}$ inocula than with un-inoculated soil, and did not differ between plant-conditioned soil inocula. Plants exposed to Meloidogyne and growing with $\mathrm{AO}, \mathrm{BH}, \mathrm{GV}$ inocula had a significantly lower proportion of yellow leaves, than plants grown with Meloidogyne and RA inoculum. In presence of Meloidogyne, none of the plantconditioned inocula were significantly different from un-inoculated soil. When exposed to Pythium, plants with $\mathrm{AO}$ and FF inocula had a significantly lower proportion of yellow leaves than plants grown in uninoculated soil, and there were no significant differences between plant-conditioned inocula (Fig. 2c).

After growth cycle 2, there were no significant interactions between any factors on shoot biomass or root biomass. Root biomass and leaf yellowness differed significantly between plant-conditioned inocula (Table 2; Fig. 3). Shoot biomass and leaf yellowness were significantly influenced by "disease treatment 1 ". Shoot biomass and leaf yellowness of plants grown in soil where Meloidogyne were added in cycle 1 was higher than in plants grown in soil from the control treatment from cycle 1. Plants growing in soil inoculated with diseased soil in cycle 2 had higher root biomass than those growing in soil from the control and the Pythium treatment. Plants exposed to Pythium in cycle 2 in general had significantly lower leaf yellowness than plants from the control treatment. Leaf yellowness varied between disease and soil treatments resulting in significant interactions between "disease treatment 1 " and "soil", "disease treatment 1 " and "disease treatment 2 ", and between "disease treatment 1 ", "disease treatment 2" and "soil" (Table 2, Fig. 3c). In the Pythium treatment in cycle 1 yellowness was higher in inoculated soil that had been conditioned by LP and RA, and lower in soil conditioned by HL. For AO, GV, and HR and un- inoculated soil, yellowness was higher in the control treatment in cycle 1 than in other disease treatments, while in cycle 2 this was true for the control treatment for soil conditioned by BH, HL and LP. Yellowness in the diseased soil treatment in cycle 2 was higher when plants were grown in soil conditioned by FF and RA. Exposure to Pythium in both cycles led to higher yellowness in RA soil, while exposure first to Meloidogyne and then Pythium led to higher yellowness in AO soil and in un-inoculated soil (Table 2; Fig. 3c). Plants grown in diseased soil had healthier roots (lower root darkness scores) than plants from the Pythium and control treatment. However, the root nematode score was significantly higher for plants inoculated with diseased soil in cycle 2 than for other plants (Table S2; Fig.S3). When un-inoculated soil was included in the same analysis, the results were similar, except that for this analysis, "disease treatment 1 " effects were significant for chrysanthemum root biomass (Table S3).

We subsequently analyzed the ten soil categories separately (the eight plant-conditioned soil inocula, the un-inoculated soil from cycle 1, and new sterile soil). Shoot biomass of plants grown in LP and un-inoculated soil from cycle 1 and in new sterile soil differed significantly between disease treatments (Fig. 3a). Root biomass of plants in all soil categories, except for HL inoculum, differed significantly between disease treatments (Fig. 3b). Overall, in soils from cycle 1 (8 plantconditioned inocula and un-inoculated soil), chrysanthemum grown in pots where diseased soil was added had more biomass than chrysanthemum exposed to the other disease treatments. At the end of cycle 2, for plants grown in un-inoculated soil from cycle 1, biomass in the double control treatment $(\mathrm{C}-\mathrm{C})$ was lower than in the other treatments. For chrysanthemum grown in sterile soil in cycle 2, addition of diseased soil led to a reduction in biomass, and Pythium addition did not affect biomass. In cycle 2 , root darkness did not differ between 

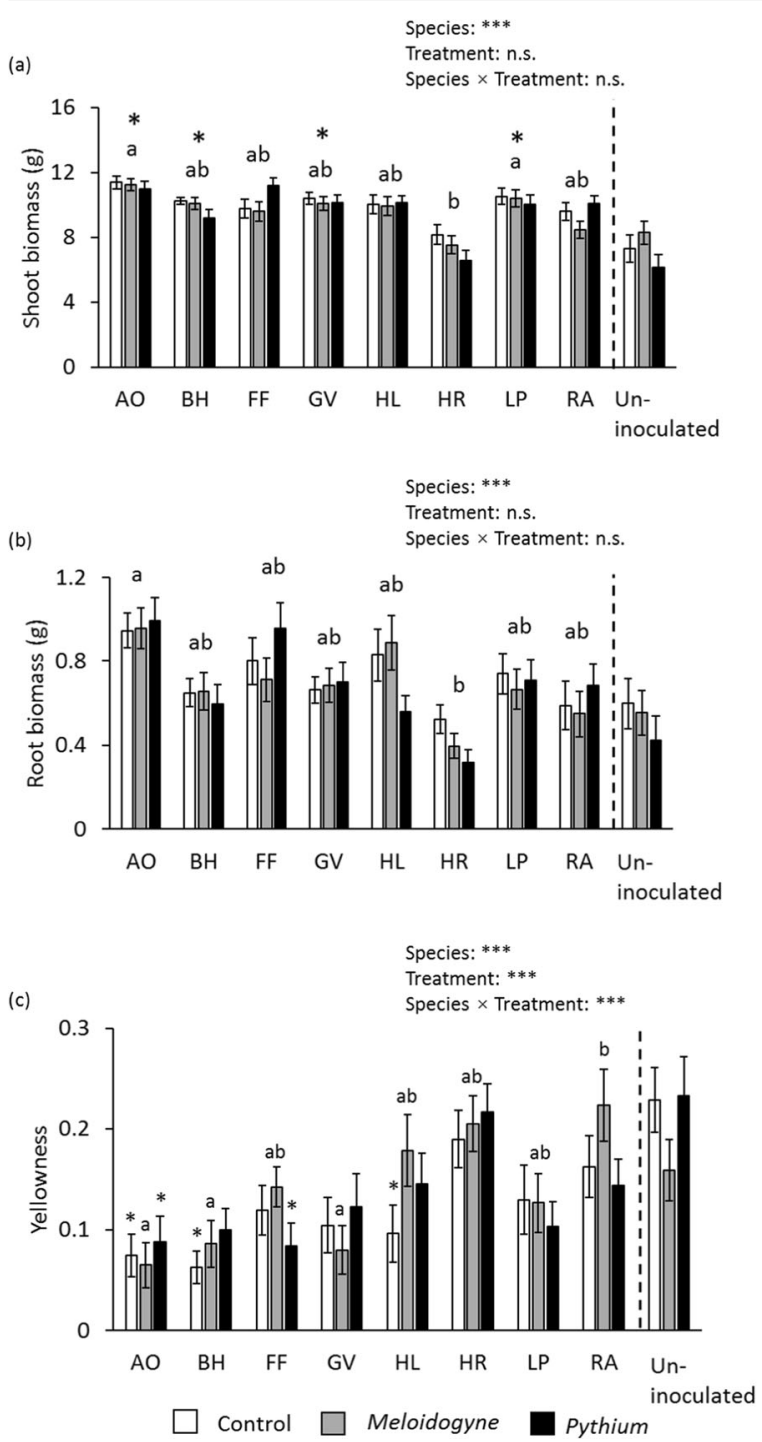

Fig. 2 Chrysanthemum shoot biomass (a), root biomass (b) and leaf yellowness (c) in plant-conditioned soil inocula with different disease treatments after growth cycle 1 . White bars indicate chrysanthemum performance in the control treatment, grey bars the Meloidogyne treatment, and black bars the Pythium treatment. Statistics presented in the upper part of each panel are the overall effects of the plant-conditioned inocula and disease treatment 1 from a mixed linear model (generalized mixed linear model for leaf yellowness). $* * *$ indicates significant difference at $P<0.001$. "n.s." indicates no significant difference detected. Different letters above each set of bars indicate significant difference between plant-conditioned soil inocula $(P<0.05)$. * indicates significant difference of a plant-conditioned inoculum and the un-inoculated soil $(P<0.05)$

disease treatments within each soil category (Fig.S3a). However, nematodes scores in plants grown in pots where diseased soil was added were much higher than in plants from other treatments (Fig.S3b). Figures and statistical analyses presenting the ten soil categories for each of the nine disease treatment combinations are shown in the appendix (Fig.S4, Table S4, S5).

In cycle 2 , on average, chrysanthemum shoot biomass and root biomass was higher in pots that were inoculated in cycle 1 with plant-conditioned soil than in pots that were not inoculated in cycle 1 (Fig. 4a, d). However, in soil where chrysanthemum had been grown before, both in pots inoculated with plant-conditioned soil and in un-inoculated soil, shoot and root biomass was lower in cycle 2 than in sterile soil for the control (Fig.4a, d) and Pythium treatment (Fig. 4b, e) in cycle 2. For plants grown in pots where diseased soil was added in cycle 2, there were no significant differences for shoot and root biomass between the three types of soils (cycle 1 inoculated, cycle 1 un-inoculated, cycle 2 sterile; Fig. 3c, f). Leaf yellowness did not significantly differ among plants grown in the different types of soils (Fig. $4 \mathrm{~g}-\mathrm{i}$ ). Moreover, there was no significant relationship between root biomass of the conditioning plant species and root biomass of chrysanthemum in both cycle 1 and cycle 2 (Fig.S5).

\section{Discussion}

Our results show that the effects of soil inoculation at the start of cycle 1 were still detectable after the second growth cycle. After two growth cycles, chrysanthemum generally grew better in new soil (sterile soil) than in soils in which chrysanthemum had been grown before (i.e. in plant-conditioned soil and in un-inoculated soil), indicating that this species exhibits a negative conspecific plant-soil feedback. Importantly, inoculating sterilized soil with plant-conditioned soil reduced this negative conspecific feedback effect, as plant growth in cycle 2 in plant-conditioned soil was better than in uninoculated soil. Moreover, in cycle 2, the effects of plant-conditioned inocula on plant growth were less variable than in cycle 1 , suggesting that growth of chrysanthemum in all soils for one cycle caused all plant-conditioned soils to develop in a similar way. Remarkably, inoculation with soil from a commercial greenhouse that contained diseases had a strong negative effect on plant growth when this was done in sterile soil, but when this "diseased soil" was added to soils in which chrysanthemum had been grown before, it increased plant growth. 
Table 2 Overall effects of plant-conditioned soil inocula, disease treatment 1 , and disease treatment 2 on chrysanthemum shoot biomass, root biomass and leaf yellowness at the end of cycle 2 . "Species" indicates the identity of plant species that conditioned the soil used for the inoculum. "Treatment 1 " indicates the disease treatments imposed during cycle 1, "Treatment 2 " indicates the disease treatments added in cycle 2. Presented are F-values obtained from a linear mixed model (generalized linear mixed model for leaf yellowness). For pairwise comparisons between each category of disease treatments T-values are presented (Z-value for leaf yellowness). *,**,*** indicates significant differences at $P<0.05,0.01$ or 0.001 , respectively

\begin{tabular}{|c|c|c|c|c|}
\hline & Df & Shoot biomass & Root biomass & Yellowness \\
\hline Species & 7,72 & 1.937 & $2.568^{*}$ & $2.147 *$ \\
\hline Treatment1 & 2576 & $3.081 *$ & 2.425 & $9.190 * *$ \\
\hline Control vs Meloidogyne & & $-2.292 *$ & -2.187 & $2.638 *$ \\
\hline Control vs Pythium & & -0.320 & -0.868 & 0.097 \\
\hline Meloidogyne vs Pythium & & 1.972 & 1.318 & 0.055 \\
\hline Treatment 2 & 2576 & 0.869 & $52.441 * * *$ & $71.139 * * *$ \\
\hline Control vs Diseased soil & & -0.101 & $-9.463 * * *$ & 0.248 \\
\hline Control vs Pythium & & -1.189 & -1.339 & $2.253^{*}$ \\
\hline Diseased soil vs Pythium & & -1.088 & $8.123 * * *$ & -0.220 \\
\hline Treatment $1 *$ Species & 14,576 & 1.155 & 0.868 & $4.401 *$ \\
\hline Treatment $2 *$ Species & 14,576 & 0.833 & 0.496 & 2.335 \\
\hline Treatment $1 *$ treatment 2 & 4576 & 0.676 & 0.671 & $8.944 *$ \\
\hline Treatment $1 *$ Treatment $2 *$ Species & 28,576 & 1.053 & 0.839 & $3.725 *$ \\
\hline
\end{tabular}

In accordance with the first hypothesis, the effects of adding inocula that consisted of soil in which another plant had been grown (plant-conditioned inocula) were still present in the second growth cycle. This is in line with other studies that show that plant-mediated changes in the soil can affect the performance of other succeeding plants in both agricultural and natural ecosystems (de la Peña et al. 2016; Jangid et al. 2011; Wubs and Bezemer 2018). In general, the mechanisms for these effects are difficult to disentangle because they can be due to changes in soil abiotic and biotic conditions (de la Peña et al. 2016). In this study, we found that addition of $10 \%$ of a soil inoculum to sterile soil influenced chrysanthemum growth during two cycles. The small amount of soil inoculum added, and the high fertilization rates in all treatments, make it unlikely that soil nutrient availability played a role, and hints at a pivotal role of the soil microbial community (Brinkman et al. 2010). Extended impacts of the soil microbial community on plant health, have also been reported in studies on soil disease suppression, where suppressiveness of the soil against pathogens could be maintained for several growth cycles (Lapsansky et al. 2016; Janvier et al. 2007; Mendes et al. 2011). In the current study, we only tested the effects of soil inoculation for two growth cycles. How long these effects of the soil inocula persist, and whether and how they influence chrysanthemum performance in subsequent growth cycles is unknown and this is an important question to be addressed in future studies.

In accordance with the second hypothesis, chrysanthemum, which was the most recently growing species in the soil, had a stronger influence on biomass in cycle 2 than the plant species that conditioned the soil that was used for inoculation at the start of cycle 1. Additionally, we also observed that chrysanthemum grew better in new sterile soil than in soil in which it had been grown before. This indicates that chrysanthemum exhibits a negative feedback, due to the build-up of pathogens in the rhizosphere or due to the release of plant metabolites in the soil that inhibit plant growth (i.e. autotoxic effects, Zhou et al. 2009). Inoculation of the sterilized soil with soil that was conditioned by another plant species reduced this negative plant-soil feedback effect. Such negative conspecific feedbacks are a commonly observed problem in continuous cropping systems (Song et al. 2013; Zhou et al. 2009; Wang et al. 2017b). However, we now show that adding an inoculum consisting of soil conditioned by other plant species to the sterilized soil before the first growth cycle starts can reduce this negative effect. Previous studies have reported that certain soil microbiomes have disease- 

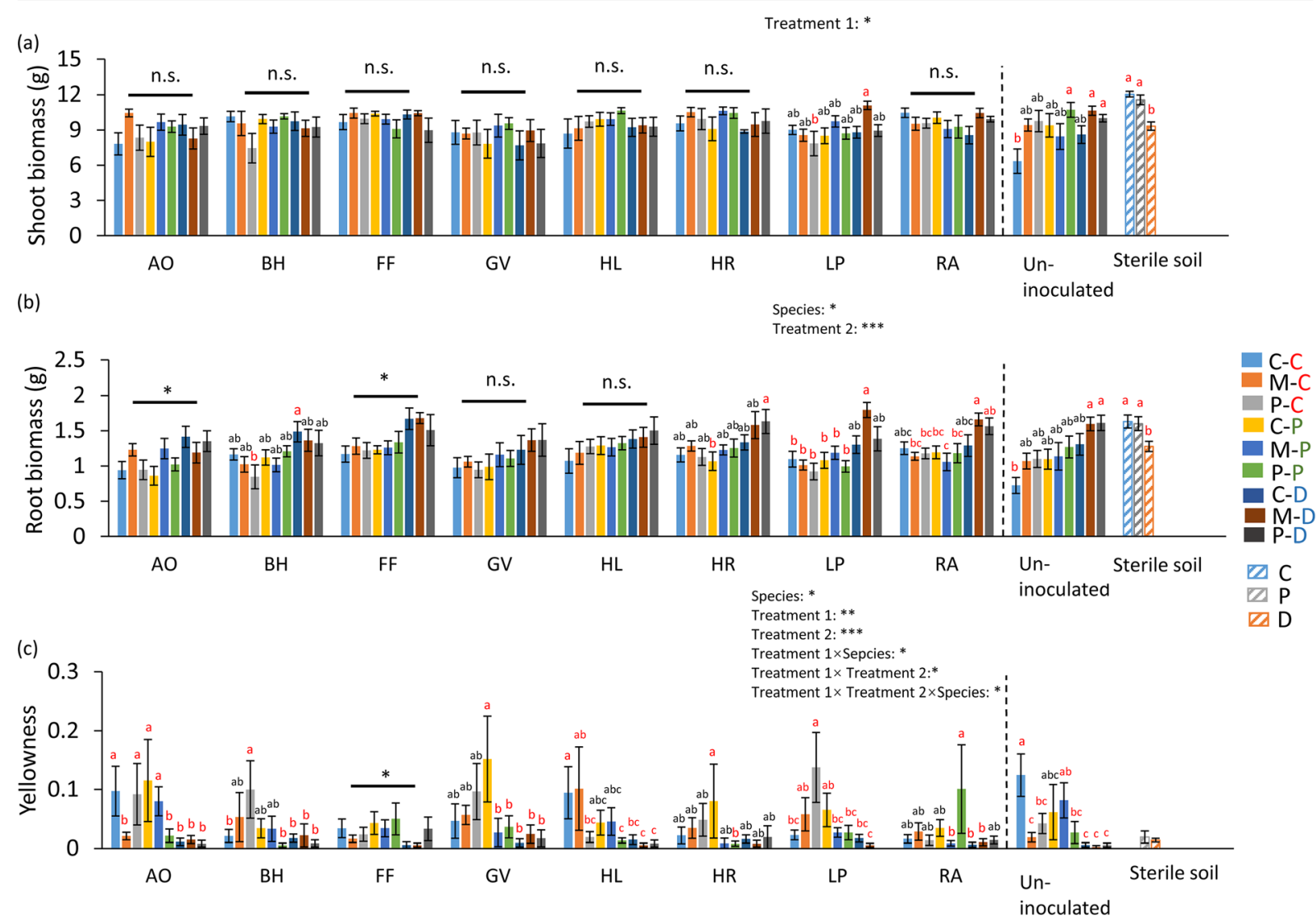

Fig. 3 Chrysanthemum shoot biomass (a), root biomass (b) and leaf yellowness (c) in plant-conditioned soil inocula with different disease treatments after growth cycle 2. Colors of the bars indicate the different inoculation and disease treatment combinations. * indicates that for this specific soil inoculum there is an overall significant effect of disease treatments, however individual treatments do not significantly differ. Statistics presented in the upper

suppressive properties in soils (Schlatter et al. 2017; Mendes et al. 2011; Ridout and Newcombe 2016; Berendsen et al. 2012). Our study further shows that over successive growth cycles, microbiomes of unrelated plant species can be used to reduce negative conspecific plant-soil feedbacks in horticultural crops. This could be achieved by changing the soil via growing another crop intermediately, or by soil inoculation, as in our study. Our study indicates that addition of soil conditioned by other plant species to sterilized or steamed soil could potentially increase crop yield of later harvests, as well as increase the number of crops that can be harvested sequentially before the soil has to be steamed again. Steaming of soil exhibits a significant environmental footprint (Ispahani et al. 2008) and an additional harvest before the grower has to steam the part of each figure are the significant effects, more details are presented in Table 2. For inocula with different letters above bars there is an overall significant effect of disease treatments and significant differences among the treatments are highlighted with red letters. "n.s." indicates no significant difference detected. Statistics of each soil category are presented in Table S4

soil again would increase the sustainability of the crop, which is an important aim in the chrysanthemum sector (Kos et al. 2014).

To answer the third hypothesis, the plant-conditioned soil inocula should be characterized as positive and negative. However, compared to control soil, in the first cycle there were only positive to neutral effects of inoculation with plant-conditioned soil, and the difference among the effects of plant-conditioned soil inocula was less variable in the second than in the first cycle. For example, in cycle 1, addition of soil conditioned by the plant species Hypochaeris radicata (HR) had the most negative influence on chrysanthemum, while inoculation with from soil conditioned by Anthoxanthum odoratum (AO) resulted in the greatest biomass. In the second cycle, this difference between $\mathrm{AO}$ and HR 

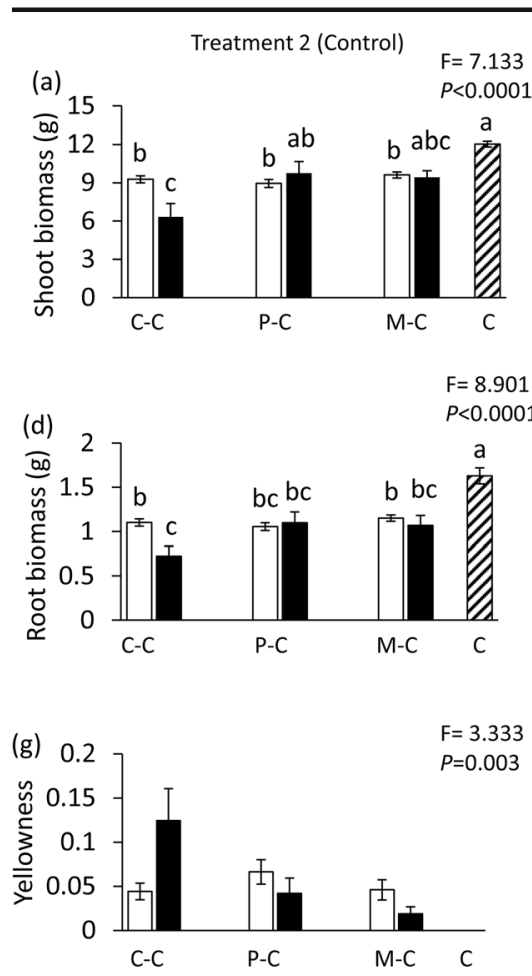

Treatment 2 (Pythium)

(b)

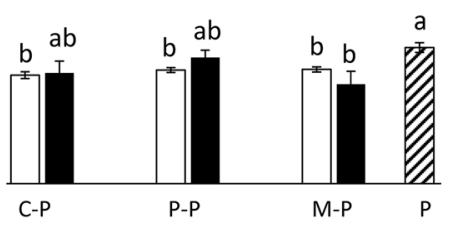

(e)

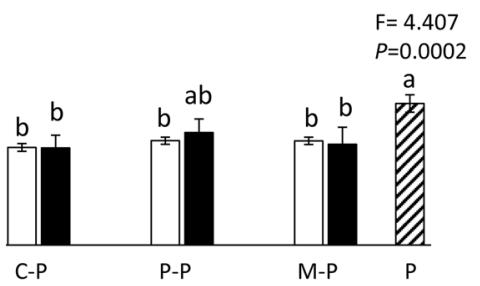

(h)

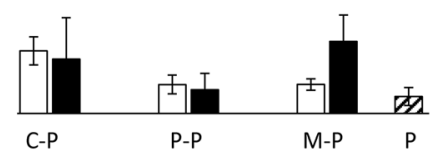

(c)

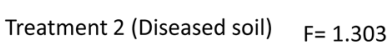

$P=0.256$

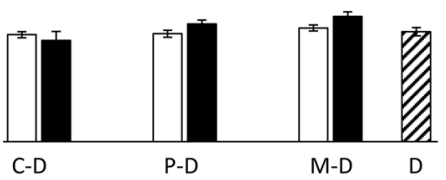

(f)

$\mathrm{F}=1.318$ $P=0.249$

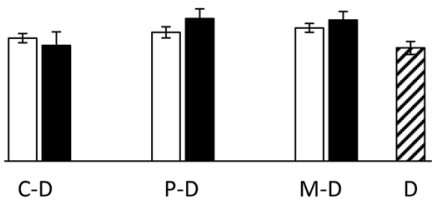

(i)

$\mathrm{F}=0.525$ $P=0.789$

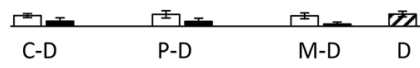

\section{Inoculated soil}

Fig. 4 Average values for inoculated soil (average of the 8 inocula), un-inoculated soil (100\% sterile in cycle 1) and new sterile soil $(100 \%$ sterile soil in cycle 2$)$ for chrysanthemum shoot biomass $(\mathbf{a}-\mathbf{c})$, root biomass $(\mathbf{d}-\mathbf{f})$ and leaf yellowness $(\mathbf{g}-\mathbf{i})$ at the end of cycle 2 . White bars indicate average chrysanthemum performance in soil inoculated with plant-conditioned inocula, black bars indicate performance in un-inoculated soil from cycle 1, striped bars indicate performance in sterile soil from cycle 2 . For

disappeared. Other studies on the temporal dynamics of plant-soil feedback effects have argued that the changes in plant-soil feedbacks over time will depend on the target plant species in the feedback phase (Kardol et al. 2006, 2013; Hawkes et al. 2013). In this study, the effects of all inocula converged over time. This is probably because of the overriding effects of chrysanthemum on the soil. We did not examine the microbial community of the different inocula and how much of these differences remained present after chrysanthemum had grown in the inoculated soils. Future studies should test how the composition of the soil microbial communities changes due to inoculation with plant-conditioned soil and how this is subsequently influenced by the growth of chrysanthemum in these soils.

In our study, addition of Pythium and Meloidogyne did not have consistent negative effects on plant growth

\section{Un-inoculated soil $\mathbb{Z}$ Sterile soil}

each plant parameter, the figure is grouped by disease treatments imposed in cycle 2 and split into three panels. In each panel, a one-way ANOVA was used to test the overall differences between all the bars, and a post hoc Tukey test was used to do pairwise comparisons between bars. Bars with identical letters are not significantly different. F-value and $P$ value of one-way ANOVAs are presented in the upper part of each panel

and health. After cycle 2, leaf yellowness differed significantly between soil treatments. However, this pattern was not consistent among the disease treatments imposed in cycle 1 or 2 . Moreover, for some soils and in both cycles, the yellowness of plants in the control treatments was significantly higher than in disease treatments, suggesting that the disease treatments may even reduce yellowness. Although leaf yellowness is a health indicator in chrysanthemum (Reddy 2015), it is not caused specifically by Pythium or Meloidogyne, and other pathogens that may have been present in the soil inocula or in the diseased soil could also cause leaf yellowing. On the basis of these results, conclusions regarding the fourth hypothesis cannot be made. The spore and juvenile density of Pythium and Meloidogyne used in this study are sufficient to cause symptoms in plant growth (van der Wurff et al. 2010) but we 
speculate that the virulence of the disease inocula was limited. This is supported by the low nematode and darkness scores in the roots and the lack of differences among disease treatments in these scores. It is also possible that the Pythium and Meloidogyne inoculations were ineffective e.g. because these pathogens did not establish successfully in the soil or were outcompeted by microbes present in the soil. An interesting finding of our study is that the addition of diseased soil in cycle 2 significantly increased performance of chrysanthemum in soils where chrysanthemum had been grown before, but that it had a negative effect on biomass when added to new sterile soil. Plant performance in the soils in which chrysanthemum had been grown before was worse than in new sterile soil. Hence, a negative treatment (i.e. diseased soil) was imposed to soils where plant performance was reduced already. This phenomenon that addition of a negative treatment to a soil which already has a negative effect is similar to a concept of soil immunity (Raaijmakers and Mazzola 2016). However, soil immunity typically appears after an outbreak of a soil-borne disease, and it takes a long period of mono-cropping, up to decades, to achieve soil immunity (Hamid et al. 2017; Raaijmakers and Mazzola 2016). Whether, the soils from cycle one in our study have become immune to soil diseases is unknown, and further work is required to disentangle these plant-soil feedback and soil disease interactions.

In conclusion, this study provides a possible application of the plant-soil feedback concept in horticulture, and demonstrates that inoculation of sterile soil with live soil conditioned by other plant species can reduce but not completely remove the negative conspecific plantsoil feedback of chrysanthemum. We did not observe strong effects of addition of soil diseases. Plant growth was best in sterile soil but, importantly, this effect disappeared when plants were grown in pots where soil was added from a commercial greenhouse with soil disease problems. Future studies should unravel the role of the composition of the microbiome, and mechanisms behind the soil inoculation effects, as well as test the effects of soil inoculation with whole microbiomes in a real horticultural greenhouse scenarios.

Acknowledgements The authors thank Manuela van Leeuwen (Dümmen Orange) for providing chrysanthemum cuttings, Rene Corsten (Delphy) for advice on optimal growing conditions of chrysanthemum and Marta Streminska (Wageningen UR Greenhouse Horticulture) for providing P. ultimum inoculum. We thank the Chinese Scholarship Council (CSC) for funding HM's research activities, and the Netherlands Organization for Scientific Research (NWO, project no. 870.15.080) for funding this research. This is publication 6601 of the Netherlands Institute of Ecology (NIOO-KNAW).

\section{References}

Alabouvette C, Olivain C, Migheli Q, Steinberg C (2009) Microbiological control of soil-borne phytopathogenic fungi with special emphasis on wilt-inducing Fusarium oxysporum. New Phytol 184:529-544

Berendsen RL, Pieterse CMJ, Bakker PAHM (2012) The rhizosphere microbiome and plant health. Trends Plant Sci 17: 478-486

Bever JD, Westover KM, Antonovics J (1997) Incorporating the soil community into plant population dynamics: the utility of the feedback approach. J Ecol 85:561-573

Bezemer TM, Jing J, Bakx-Schotman JMT, Bijleveld E (2018) Plant competition alters the temporal dynamics of plant-soil feedbacks. J Ecol (in press)

Brinkman EP, van der Putten WH, Bakker E, Verhoeven KJF (2010) Plant-soil feedback: experimental approaches, statistical analyses and ecological interpretations. J Ecol 98:1063-1073

Castillo P, Mora-Rodríguez MP, Navas-Cortés JA, Jiménez-Díaz RM (1998) Interactions of Pratylenchus thornei and Fusarium oxysporum f. sp. ciceris on chickpea. Phytopathology 88:828-836

Chaparro JM, Sheflin AM, Manter DK, Vivanco JM (2012) Manipulating the soil microbiome to increase soil health and plant fertility. Biol Fertil Soils 48:489-499

Cortois R, Schröder-Georgi T, Weigelt A, van der Putten WH, De Deyn GB (2016) Plant-soil feedbacks: role of plant functional group and plant traits. J Ecol 104:1608-1617

de la Peña E, Baeten L, Steel H, Viaene N, De Sutter N, De Schrijver A, Verheyen K (2016) Beyond plant-soil feedbacks: mechanisms driving plant community shifts due to land-use legacies in post-agricultural forests. Funct Ecol 30: 1073-1085

Detheridge AP, Brand G, Fychan R, Crotty FV, Sanderson R, Griffith GW, Marley CL (2016) The legacy effect of cover crops on soil fungal populations in a cereal rotation. Agric Ecosyst Environ 228:49-61

Dhandaydham M, Charles L, Zhu H, Starr JL, Huguet T, Cook DR, Prosperi J, Opperman C (2008) Characterization of rootknot nematode resistance in Medicago truncatula. J Nematol 40:46-54

Gómez Expósito R, Bruijn ID, Postma J, Raaijmakers JM (2017) Current insights into the role of rhizosphere bacteria in disease suppressive soils. Front Microbiol 8:2529

Hajihassani A, Maafi ZT, Hosseininejad A (2013) Interactions between Heterodera filipjevi and Fusarium culmorum, and between $H$. filipjevi and Bipolaris sorokiniana in winter wheat. J Plant Dis Protect 120:77-84

Hamid MI, Hussain M, Wu Y, Zhang X, Xiang M, Liu X (2017) Successive soybean-monoculture cropping assembles 
rhizosphere microbial communities for the soil suppression of soybean cyst nematode. Front Microbiol 93:1

Hawkes CV, Kivlin SN, Du J, Eviner VT (2013) The temporal development and additivity of plant-soil feedback in perennial grasses. Plant Soil 369:141-150

Huang L, Song L, Xia X, Mao W, Shi K, Zhou Y, Yu J (2013) Plant-soil feedbacks and soil sickness: from mechanisms to application in agriculture. J Chem Ecol 39:232-242

Ispahani SK, Goud JC, Termorshuizen AJ, Morton A, Barbara DJ (2008) Host specificity, but not high-temperature tolerance, is associated with recent outbreaks of Verticillium dahliae in chrysanthemum in the Netherlands. Eur J Plant Pathol 122: 437-442

Jangid K, Williams MA, Franzluebbers AJ, Schmidt TM, Coleman DC, Whitman WB (2011) Land-use history has a stronger impact on soil microbial community composition than aboveground vegetation and soil properties. Soil Biol Biochem 43:2184-2193

Janvier C, Villeneuve F, Alabouvette C, Edel-Hermann V, Mateille T, Steinberg C (2007) Soil health through soil disease suppression: which strategy from descriptors to indicators? Soil Biol Biochem 39:1-23

Johnson AW, Littrell RH (1970) Pathogenicity of Pythium aphanidermatum to chrysanthemum in combined inoculations with Belonolaimus longicaudatus or Meloidogyne incognita. J Nematol 2:255

Kardol P, Bezemer TM, van der Putten WH (2006) Temporal variation in plant-soil feedback controls succession. Ecol Lett 9:1080-1088

Kardol P, Deyn GB, Laliberte E, Mariotte P, Hawkes CV (2013) Biotic plant-soil feedbacks across temporal scales. J Ecol 101:309-315

Khan M, Siddiqui ZA (2017) Effect of simultaneous and sequential inoculations of Meloidogyne incognita, Ralstonia solanacearum and Phomopsis vexans on eggplant in sand mix and fly ash mix soil. Phytoparasitica 45:599-609

Kos SP, Klinkhamer PGL, Leiss KA (2014) Cross-resistance of chrysanthemum to western flower thrips, celery leafminer, and two-spotted spider mite. Entomol Exp Appl 151: 198-208

Kulmatiski A, Beard KH (2011) Long-term plant growth legacies overwhelm short-term plant growth effects on soil microbial community structure. Soil Biol Biochem 43:823-830

Kulmatiski A, Beard KH, Stark JM (2006) Soil history as a primary control on plant invasion in abandoned agricultural fields. J Appl Ecol 43:868-876

Kulmatiski A, Beard KH, Stevens JR, Cobbold SM (2008) Plant-soil feedbacks: a meta-analytical review. Ecol Lett 11:980-992

Lapsansky ER, Milroy AM, Andales MJ, Vivanco JM (2016) Soil memory as a potential mechanism for encouraging sustainable plant health and productivity. Curr Opin Biotechnol 38: 137-142

Lennon JT, Jones SE (2011) Microbial seed banks: the ecological and evolutionary implications of dormancy. Nat Rev Microbiol 9:119-130

Ma H, Pineda A, van der Wurff AWG, Raaijmakers C, Bezemer TM (2017) Plant-soil feedback effects on growth, defense and susceptibility to a soil-borne disease in cut flower crop: species and functional group effects. Front Plant Sci 8:2127
Mallon CA, Le Roux X, van Doorn GS, Dini-Andreote F, Poly F, Salles JF (2018) The impact of failure: unsuccessful bacterial invasions steer the soil microbial community away from the invader's niche. ISME J 12:728-741

Mariotte P, Mehrabi Z, Bezemer TM, De Deyn GB, Kulmatiski A, Drigo B, Ciska Veen GF, van der Heijden MGA, Kardol P (2017) Plant-soil feedback: bridging natural and agricultural sciences. Trends Ecol Evol 33:129-142

Mendes R, Kruijt M, De Bruijn I, Dekkers E, van der Voort M, Schneider JHM, Piceno YM, DeSantis TZ, Andersen GL, Bakker PAHM, Raaijmakers JM (2011) Deciphering the rhizosphere microbiome for disease-suppressive bacteria. Science 332:1097-1100

Pettitt TR, Wainwright MF, Wakeham AJ, White JG (2011) A simple detached leaf assay provides rapid and inexpensive determination of pathogenicity of Pythium isolates to 'all year round'(AYR) chrysanthemum roots. Plant Pathol 60: 946-956

Pineda A, Kaplan I, Bezemer TM (2017) Steering soil microbiomes to suppress aboveground insect pests. Trends Plant Sci 22:770-778

Pung SH, Sivasithamparam K, Barbetti MJ (1991) Effect of placement and sequence of inoculation with Fusarium oxysporum on its interaction with Meloidogyne arenaria on subterranean clover. J Phytopathol 132:273-286

R Core Team (2017) R: A language and environment for statistical computing. R Foundation for Statistical Computing, Vienna, Austria. URL https://www.R-project.org/

Raaijmakers JM, Mazzola M (2016) Soil immune responses. Science 352:1392-1393

Reddy PP (2015) Sustainable crop protection under protected cultivation. Springer, Singapore, pp 368-371

Ridout M, Newcombe G (2016) Disease suppression in winter wheat from novel symbiosis with forest fungi. Fungal Ecol 20:40-48

Schlatter D, Kinkel L, Thomashow L, Weller D, Paulitz T (2017) Disease suppressive soils: new insights from the soil microbiome. Phytopathology 107:1284-1297

Siddiqui ZA, Mir RA, Mahmood I (1999) Effects of Meloidogyne incognita, Fusarium oxysporum f. sp. pisi, Rhizobium sp., and different soil types on growth, chlorophyll, and carotenoid pigments of pea. Isr J Plant Sci 47:251-256

Siddiqui Y, Ali A, Naidu Y (2014) Histopathological changes induced by Meloidogyne incognita in some ornamental plants. Crop Prot 65:216-220

Song A, Zhao S, Chen S, Jiang J, Chen S, Li H, Chen Y, Chen X, Fang W, Chen F (2013) The abundance and diversity of soil fungi in continuously monocropped chrysanthemum. Sci World J 2013:632920

Tamm L, Thürig B, Bruns C, Fuchs JG, Köpke U, Laustela M, Leifert C, Mahlberg N, Nietlispach B, Schmidt C, Weber F, Fließbach A (2010) Soil type, management history, and soil amendments influence the development of soil-borne (Rhizoctonia solani, Pythium ultimum) and air-borne (Phytophthora infestans, Hyaloperonospora parasitica) diseases. Eur J Plant Pathol 127:465-481

Thuerig B, Fließbach A, Berger N, Fuchs JG, Kraus N, Mahlberg N, Nietlispach B, Tamm L (2009) Reestablishment of suppressiveness to soil- and air-borne diseases by re-inoculation of soil microbial communities. Soil Biol Biochem 41:2153-2161 
van de Voorde TF, van der Putten WH, Bezemer TM (2011) Intra-and interspecific plant-soil interactions, soil legacies and priority effects during old-field succession. J Ecol 99:945-953

van der Gaag DJ, Wever G (2005) Conduciveness of different soilless growing media to Pythium root and crown rot of cucumber under nearcommercial conditions. Eur J Plant Pathol 112:31-41

van der Putten WH, Bardgett RD, Bever JD, Bezemer TM, Casper BB, Fukami T, Kardol P, Klironomos JN, Kulmatiski A, Schweitzer JA, Suding KN, Van de Voorde TFJ, Wardle DA (2013) Plant-soil feedbacks: the past, the present and future challenges. J Ecol 101: 265-276

van der Wurff AWG, van Slooten MA, Hamelink R, Böhne S, van Wensveen W (2010) Soil suppressiveness towards Meloidogyne, Verticillium or Pythium in greenhouse horticulture. ISHS Acta Hortic 915:141-149

Wang GZ, Li HG, Christie P, Zhang FS, Zhang JL, Bever JD (2017a) Plant-soil feedback contributes to intercropping overyielding by reducing the negative effect of take-all on wheat and compensating the growth of faba bean. Plant Soil 415:1-12

Wang J, Li X, Xing S, Ma Z, Hu S, Tu C (2017b) Bio-organic fertilizer promotes plant growth and yield and improves soil microbial community in continuous monoculture system of Chrysanthemum morifolium cv. Chuju. Int J Agric Biol 19: 563-568

Wardle DA, Bardgett RD, Klironomos JN, Setälä H, van der Putten WH, Wall DH (2004) Ecological linkages between aboveground and belowground biota. Science 304:1629-1633

Whitelaw-Weckert MA, Rahman L, Appleby LM, Hall A, Clark AC, Waite H, Hardie WJ (2013) Co-infection by Botryosphaeriaceae and Ilyonectria spp. fungi during propagation causes decline of young grafted grapevines. Plant Pathol 62:1226-1237

Wubs ERJ, Bezemer TM (2018) Temporal carry-over effects in sequential plant-soil feedbacks. Oikos 127:220-229

Wurst S, Ohgushi T (2015) Do plant-and soil-mediated legacy effects impact future biotic interactions? Funct Ecol 29: 1373-1382

Zhou K, Zhou X, Yang L, Hao F, Wang Z, Guo W (2009) Autotoxic effects of Chrysanthemum (Chrysanthemum morifolium (Ramat) Tzvel.). Allelopathy J 24:91-102

Zhou X, Liu J, Wu F (2017) Soil microbial communities in cucumber monoculture and rotation systems and their feedback effects on cucumber seedling growth. Plant Soil 415: 507-520 